\title{
Emotionale Kompetenzen im Pflegeberuf - Ein trainierbarer Überlastungsschutz
}

\author{
Marcus Eckert • Melanie Schwarzbach · Torsten Tarnowski • Bernhard Sieland
}

Online publiziert: 24. Oktober 2012

(C) Springer-Verlag Wien 2012

Die Belastung von Pflegekräften in Deutschland wie auch international ist gravierend: Ein integraler Bestandteil ihrer Arbeit ist die Emotions- und Gefühlsarbeit [5, 6]. Darunter versteht man das Zeigen geforderter Emotionen, wenn diese in der entsprechenden Situation nicht erlebt werden [4]. Ein weiterer Belastungsfaktor besteht darin, emotional schwierige Situationen so zu verarbeiten, dass ein konstruktiver Lernprozess stattfinden kann. Dass dies nicht immer gelingt, zeigt sich an hohen Burnout-Zahlen im Pflegeberuf [1, 2, 7] sowie deutlich erhöhten Zahlen innerer oder tatsächlicher Kündigungen [3]. Damit wird die Belastung im Pflegeberuf nicht mehr zu einem privaten Problem der Betroffenen, sondern sie stellt eine reale wirtschaftliche Gefahr dar.

Emotionale Kompetenzen haben sich in verschiedenen längsschnittlichen Studien als guter Überlastungsschutz erwiesen: Menschen, deren emotionale Fähigkeiten und Fertigkeiten hoch ausgeprägt sind, bewältigen Beanspruchungssituationen deutlich besser als Menschen mit niedriger Ausprägung. Dass emotionale Kompetenzen zu den trainierbaren personalen Kompetenzen gehören, birgt die Chance, bereits während der Aus- aber auch in der Weiterbildung einen wirksamen Überlastungsschutz zu implementieren. Somit kann nicht nur das menschliche Leid, das mit den Folgen zu hoher Belastung einhergeht, reduziert, sondern auch die wirtschaftlichen Folgen nachhaltig eingedämmt werden. Infolgedessen ist die Stärkung personaler Ressourcen das unbedingte Gegenstück zur Verbesserung organisationaler Strukturen im Gesundheitswesen.

\section{Literatur}

1. Aiken LH, Clarke SP, Sochalski J, Busse R, Clarke H, Giovannetti $\mathrm{P}$, et al. Nurses' report on hospital care in five countries. Health Aff. 2001;20(3):43-53.

2. Bauer J, Häfner S, Kächele H, Dahlbender RW. Burnout und Wiedergewinnung seelischer Gesundheit am Arbeitsplatz. 2002. http://apdeba.org/images/stories/Biblioteca/ComisionInvestigacion/ALEMAN/burnout. Zugegriffen: 11. April 2011.

3. Hasselhorn H-M, Müller BH, Tackenberg P, Kümmerling A, Simon M. Berufsausstieg bei Pflegepersonal. Bundesanstalt für Arbeitsschutz und Arbeitsmedizin. Bremerhaven: Wirtschaftsverlag NW; 2005.

4. Hochschild AR. Das gekaufte Herz. Zur Kommerzialisierung der Gefühle. Frankfurt a. M.: Campus Verlag GmbH; 1990.

5. Neumann-Poensch S, Höller A. Gefühlsarbeit in Pflege und Betreuung. Wien: Springer; 2011.

6. Schwarz R. Supervision und professionelles Handeln Pflegender. Wiesbaden: VS Verlag für Sozialwissenschaften; 2009.

7. Van Der Schoot E, Oginska H, Estryn-Behar M, NEXT-Studiengruppe. Burnout im Pflegeberuf in Europa. In: Bundesanstalt für Arbeitsschutz und Arbeitsmedizin, Herausgeber. Berufsausstieg bei Pflegepersonal. Bremerhaven: Verlag für neue Wissenschaft GmbH; 2005. S. 57-62. 